\title{
Mechanism and Control Parameters of the Coupled Structural and Metal-Insulator Transition in Nickelates
}

\author{
Oleg E. Peil, ${ }^{1,2}$ Alexander Hampel, ${ }^{3}$ Claude Ederer, ${ }^{3}$ and Antoine Georges ${ }^{2,4,5,6}$ \\ ${ }^{1}$ Materials Center Leoben Forschung GmbH, Roseggerstraße 12, A-8700 Leoben, Austria \\ ${ }^{2}$ DQMP, Université de Genève, 24 quai Ernest Ansermet, CH-1211 Genève, Suisse \\ ${ }^{3}$ Materials Theory, ETH Zürich, Wolfgang-Pauli-Strasse 27, 8093 Zürich, Switzerland \\ ${ }^{4}$ Collège de France, 11 place Marcelin Berthelot, 75005 Paris, France \\ ${ }^{5}$ Center for Computational Quantum Physics, Flatiron Institute, 162 Fifth avenue, New York, NY 10010, USA \\ ${ }^{6}$ CPHT, Ecole Polytechnique, CNRS, Université Paris-Saclay, 91128 Palaiseau, France
}

(Dated: June 28, 2021)

\begin{abstract}
Rare-earth nickelates exhibit a remarkable metal-insulator transition accompanied by a symmetrylowering lattice distortion. Using model considerations and first-principles calculations, we present a theory of this phase transition which reveals the key role of the coupling between electronic and lattice instabilities. We show that the transition is driven by the proximity to an instability toward electronic disproportionation which couples to a specific structural distortion mode, cooperatively driving the system into the insulating state. This allows us to identify two key control parameters of the transition: the susceptibility to electronic disproportionation and the stiffness of the lattice mode. We show that our findings can be rationalized in terms of a Landau theory involving two coupled order parameters, with general implications for transition-metal oxides.
\end{abstract}

The coupling of electrons to lattice degrees of freedom provides a key opportunity to control the properties of strongly correlated materials, as in, e.g., epitaxial heterostructures 11. Such a coupling often leads to concomitant electronic and structural transitions, which have been observed in $\mathrm{V}_{2} \mathrm{O}_{3}$ [2, manganates [3], $\mathrm{Ca}_{2} \mathrm{RuO}_{4}$ [4, 5], etc. Rare-earth nickelates $\left(\mathrm{RNiO}_{3}\right)$ [68. represent an ideal playground in this respect, because their metal-insulator transition (MIT), tightly associated with a lattice mode, is easily tunable [9, 10].

The MIT in $R \mathrm{NiO}_{3}$ is accompanied by a bond disproportionation (BD), i.e., a coherent contraction of the $\mathrm{NiO}_{6}$ octahedra on one sublattice [short-bond (SB) octahedra] and an expansion of the octahedra on the other sublattice [long-bond (LB) octahedra], also referred to as the "breathing mode" (BM) [7, 11. The resulting "bonddisproportionated insulator" (BDI) is also characterized by an electronic disproportionation (ED), whereby the local configuration of SB octahedra is close to to $d^{8} \underline{L}^{2}$ and that of LB ones to $d^{8}$ [12 [15, or in terms of "frontier" $e_{g}$ orbitals to $e_{g}^{0}$ and $e_{g}^{2}$, respectively [16-19]. The electron localization on the LB sublattice is the result of a "site-selective Mott transition" [20, occurring irrespective of the (ground state) magnetic ordering for all systems with $R$-cations smaller than $\mathrm{Nd}$, and lowering the energy of the insulating phase below that of the metallic phase [14, 21, 22]. Thereby, magnetic order seems to play only a secondary role, enhancing an already existing tendency towards the MIT [23 25].

The mechanism of the interplay between electronic and lattice degrees of freedom is not yet understood. This question is of key importance to identify the driving force responsible for the $\mathrm{BD}$ and for the first-order transition [26] into the paramagnetic (PM) insulating state. This transition was previously described either as a pure charge-order transition [27] or as a result of the coupling between lattice modes only [28. Recently, the authors of Ref. [29] proposed that the transition corresponds to the gradual softening of the BM mode, associated with the opening of a Peierls gap at the Fermi level, where they used density functional theory (DFT) calculations including the $+U$ correction. However, this theory describes the transition as second order, in contradiction with experimental observations [26, 30], and furthermore cannot describe the MIT into the PM state, since $\mathrm{DFT}+U$ requires a magnetically ordered state to produce an insulating gap 31, 32. Crucially, in the absence of magnetic ordering, the Peierls gap does not open at the Fermi level [17, 33] and thus cannot be responsible for the insulating nature of the PM phase.

Here, we present a theory describing the interplay between the electronic and structural aspects of the MIT. We show that the MIT is driven by the proximity to a spontaneous ED, which leads to a strongly non-linear electronic response with respect to variations of the BM amplitude, resulting in a first-order phase transition. Our theory also identifies the BM stiffness and the electronic susceptibility at $\mathbf{q}=\left(\frac{1}{2}, \frac{1}{2}, \frac{1}{2}\right)$ as key parameters controlling the transition. Experimentally, these parameters can be tuned by the choice of the $R$ cation [6], or by epitaxial strain in thin films and heterostructures [9, 10, 34, 35]. We validate our theory by performing combined DFT and dynamical mean-field theory (DMFT) [36, 37] calculations, allowing us to explore the trends across the rare-earth series. We also rationalize the overall physical picture in terms of a Landau theory involving two coupled order parameters: the ED and an order parameter associated with the metallicity of the system.

Model description. We start by constructing a simplified model which reproduces the main features of 
the MIT in $R \mathrm{NiO}_{3}$. The model retains only the key low-energy degrees of freedom: the interacting electrons in the two frontier $e_{g}$ orbitals and the BM amplitude $Q$. The purely electronic part of the Hamiltonian, $H_{\text {band }}+H_{\text {int }}$, consists of a simplified tight-binding (TB) model: $\quad H_{\text {band }}=-\sum_{i, j, m, m^{\prime}, \sigma} t_{i j}^{m m^{\prime}} d_{i m \sigma}^{\dagger} d_{j m \sigma}$ and a local interaction term $H_{\text {int }}$. Here, $i, j$ indicate sites within a simple cubic lattice, $m=1,2$ correspond to the $d_{x^{2}-y^{2}}, d_{z^{2}}$ orbitals on each site, and hopping matrices $t_{i j}^{m m^{\prime}}$ are obtained using the Slater-Koster construction with two hopping amplitudes, $t$ and $t^{\prime}$, limited to nearest-neighbor and next-nearest neighbor sites, respectively [38]. The interaction term $H_{\text {int }}$ involves two coupling constants: a repulsive interaction $U$ and an intra-atomic (Hund's) exchange $J$, and takes the standard 2-orbital Hubbard-Kanamori form [39]. The purely lattice part is described by an elastic term: $H_{\text {latt }}=\frac{K}{2} Q^{2}$, with $K$ being the stiffness of the BM. Finally, and importantly, the coupling of the BM amplitude to the electrons is captured by a term: $H_{\mathrm{e}-\mathrm{l}}=\frac{1}{2} \sum_{m \sigma} \Delta_{m}^{s}[Q]\left[\sum_{i \in \mathrm{SB}} \hat{n}_{i m \sigma}-\sum_{i \in \mathrm{LB}} \hat{n}_{i m \sigma}\right]$, where $\hat{n}_{i m \sigma}=d_{i m \sigma}^{\dagger} d_{i m \sigma}$ is the electron occupation operator and $\Delta_{m}^{s}[Q]$ is a (Peierls-like) modulation of the on-site potential seen by orbital $m$ due to the BM structural distortion parametrized by $Q$. It couples to the operator measuring the ED between the LB and SB octahedra. The total Hamiltonian thus reads: $H=H_{\text {band }}+H_{\text {int }}+H_{\mathrm{e}-1}+H_{\text {latt }}$.

At this stage, we define the amplitude $Q$ as the disproportionation in octahedral bond lengths $b=b_{0}+Q / 2$ for LB and $b=b_{0}-Q / 2$ for SB octahedra. The modulation of the on-site potential, $\Delta_{m}^{s}[Q]$, is given by the difference between the on-site energies of the SB and LB sites: $\Delta_{m}^{s}[Q]=\varepsilon_{m}\left[b_{0}-Q / 2\right]-\varepsilon_{m}\left[b_{0}+Q / 2\right] \approx\left(d \varepsilon_{m} / d b\right)_{b_{0}} Q \equiv$ $g_{m} Q$, where we have expanded in $Q$ and introduced the electron-lattice coupling parameter $g_{m}$. As emphasized in Refs. [17, 18, an appropriate low-energy description of the "negative charge-transfer" character of $R \mathrm{NiO}_{3}$ and of their tendency to form a BDI state is obtained with $U-3 J \lesssim \Delta_{s}$. In this regime, the orbital polarization is strongly suppressed implying that the on-site energies are to a good approximation orbital-independent: $\varepsilon_{z^{2}} \approx \varepsilon_{x^{2}-y^{2}}$. We thus assume that the $e_{g}$ states are degenerate and omit the index $m$ in one-electron quantities (i.e., $g_{m}=g, \Delta_{m}^{s}=\Delta_{s}$ ) [40].

Minimizing the total energy, $E=\langle H\rangle \equiv E_{\mathrm{el}}[\nu]-$ $g Q \nu / 2+K Q^{2} / 2$, with respect to $Q$ yields:

$$
\frac{2 K}{g} \bar{Q}=\nu[\bar{Q}]
$$

where $\nu[Q]=\left\langle\hat{n}_{\mathrm{LB}}-\hat{n}_{\mathrm{SB}}\right\rangle_{Q}$ is the average ED for a given amplitude $Q$. Eq. (1) is the central equation of this article: It enables one to determine the equilibrium BM amplitude from the knowledge of the electronic response encoded in $\nu[Q]$ for a given lattice stiffness, $K$, and

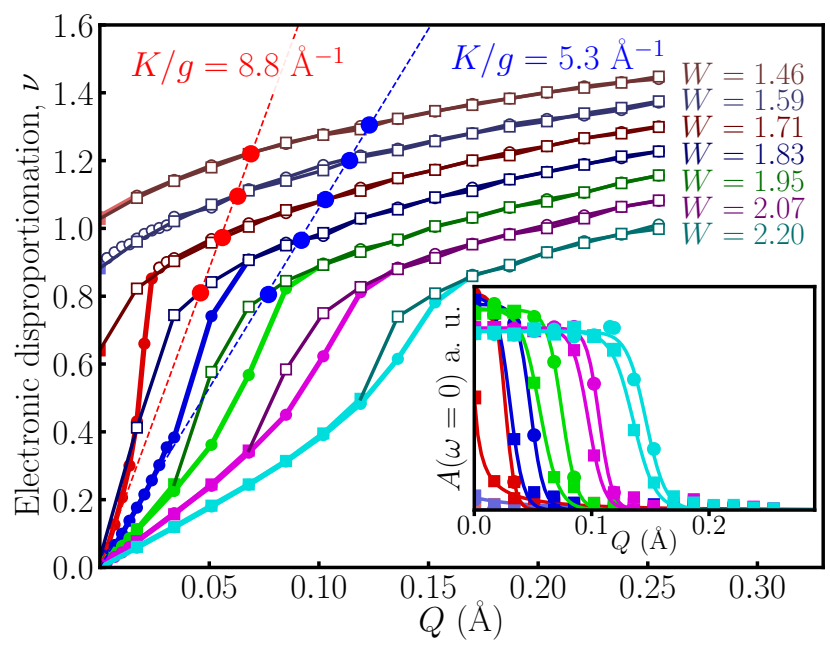

FIG. 1. Electronic disproportionation $\nu$ of the TB model as a function of bond disproportionation $Q$ for various values of the bandwidth $W$ (in eV). Open and filled symbols correspond to insulating and metallic branches, respectively. The dashed lines represent $(2 K / g) Q$ for two values of $K / g$, the intersection points giving the solutions to Eq. (1). Inset: Spectral weight as a function of $Q$.

electron-lattice coupling, $g$. The stability of the solutions of this equation is determined by the renormalized stiffness $\kappa \equiv \frac{\partial^{2} E}{\partial Q^{2}}=K-\frac{g^{2}}{2} \frac{\partial \nu}{\partial \Delta^{s}}$. Assuming no spontaneous $\operatorname{ED}(\nu[Q=0]=0)$, we obtain $\kappa=K-\chi_{e} g^{2} / 2$, where $\chi_{e} \equiv\left(\partial \nu / \partial \Delta^{s}\right)_{Q=0}$ is the electronic susceptibility associated with a "charge" modulation at a wave-vector $\mathbf{q}=\left(\frac{1}{2}, \frac{1}{2}, \frac{1}{2}\right)$ [41. Hence, the (linear) stability of the high-symmetry phase is controlled by the electronic response, $\chi_{e}$, which must be compared to $2 \mathrm{~K} / \mathrm{g}^{2}$.

We investigate the solutions to Eq. (1) by performing DMFT calculations to obtain $\nu[Q]$ for various values of the bandwidth, $W$ (see [41 for details). The results are shown in Fig. 1. The calculations were performed first for increasing values, then for decreasing values of $Q$, which resulted in a hysteresis. The most important feature of this plot is the strongly non-linear dependence of $\nu$ on $Q$. Solutions of Eq. (1) are obtained by intersecting $\nu[Q]$ with the straight line $(2 \mathrm{~K} / \mathrm{g}) \mathrm{Q}$.

This non-linear shape of the $\nu[Q]$-curves plays an important role in determining the nature of the transition. For a given value of $K / g$, and depending on $W$, there are either one, two, or three intersection points (disregarding symmetry-equivalent solutions for $Q<0$ ). Consider $K / g=5.3 \AA^{-1}$. If $W$ is large $(W \geq 2.07 \mathrm{eV}$ in Fig. 1) the straight line intersects the ED curve only at $Q=0$, rendering it the only solution to Eq. (1). As $W$ is decreased, it reaches a value below which there are three intersection points $(W=1.95 \mathrm{eV})$. The middle intersection point corresponds to an unstable solution $(\kappa<0)$. The two remaining stable solutions, $Q=0$ and $Q=\bar{Q}[K, W]>0$ mark a coexistence of two different 


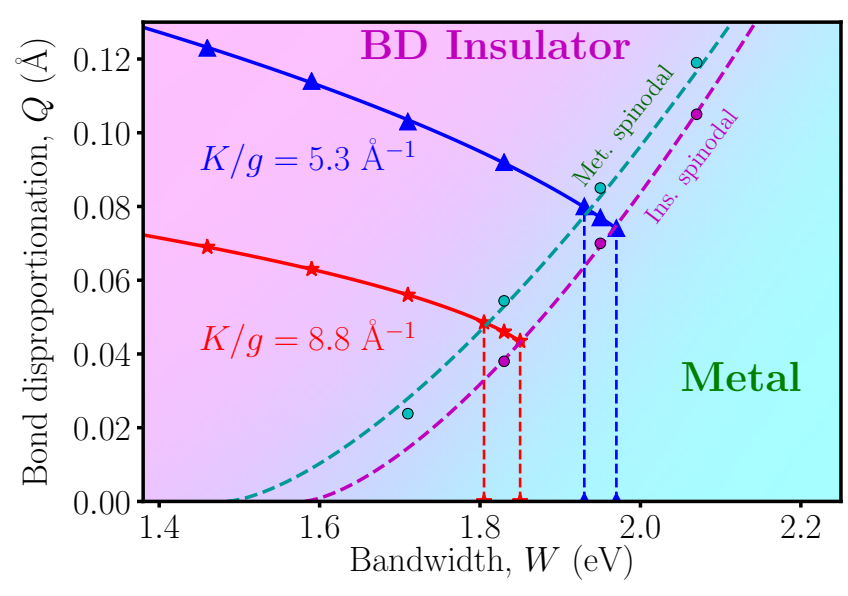

FIG. 2. Phase diagram of the TB model as a function of bandwidth $W$ and $\operatorname{BD} Q$. Circles are critical points for the metallic and insulating phases; dashed curves are spinodal lines obtained as fits to $\alpha\left(W-W_{c}\right)^{\frac{3}{2}}$ (see Landau theory). The solid lines with triangles and stars display the stable equilibrium values of $Q$ for two values of $K / g$. Vertical dashed lines designate lower and upper critical values of $W$ for the structural transition.

phases. At even smaller values of $W(W \lesssim 1.8)$ the solution at $Q=0$ gets destabilized because $\kappa[Q=0]<0$. We are then left with only one solution $Q=\bar{Q}[K, W]>0$, telling us that only the BD phase is stable here. The behavior of the solutions of Eq. (1) as a function of $W$ tells us that the BD transition is first-order. Importantly, this is not directly related to the hysteretic behavior of $\nu[Q]$. Even if there was no such hysteresis, the particular non-linear dependence of $\nu[Q]$ would imply that the BD transition is first-order. The inset of Fig. 1 displays the spectral weight at the Fermi level as a function of $Q$. We see that the system undergoes a transition from metallic to insulating behaviour as $Q$ is increased and that the transition regime corresponds to the strongly non-linear regime of $\nu[Q]$. Hence, it is the MIT which is responsible for the strong non-linearity of $\nu[Q]$.

We can now discuss the control parameters of the combined BD/MIT. Eq. (1) shows that the transition behavior depends on parameters $g, K$, and $\chi_{e}$. Our DFT calculations reveal that $g$ and $K$ vary little across the nickelate series (see [41]). Hence, for bulk $R \mathrm{NiO}_{3}$, it is the variation of the electronic susceptibility $\chi_{e}$ which plays the key role. The latter is affected by changes in the bandwidth and/or bond angles [27]. In heterostructures and under strain, the BM stiffness, $K$, varies, while $g$ remains unaffected [35. $K$ is thus likely to be an important control parameter in those cases. This may shed light on the results of Ref. 42] and motivates the variation of $K / g$ in our model calculations.

The effects of the two control parameters $K$ and $\chi_{e}$ (tuned via $K / g$ and $W$, respectively) are summarized in Fig. 2 The dashed curves indicate the boundaries

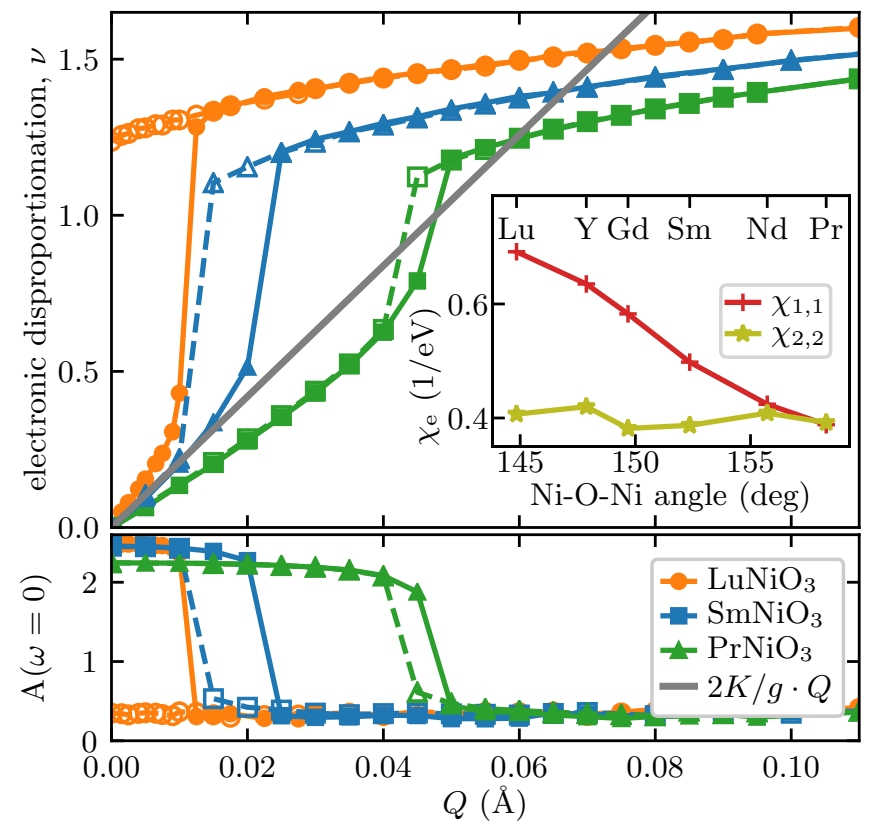

FIG. 3. Top: ED $\nu$ as a function of increasing $Q$ (filled symbols) and decreasing $Q$ (open symbols) for $R=\mathrm{Lu}$ (red), Sm (blue) and $\operatorname{Pr}$ (green). The gray line represents $(2 K / g) Q$ with values $g=3.8 \mathrm{eV} / \AA$ and $K=39.3 \mathrm{eV} / \AA^{2}$ extracted from DFT. Inset: Electronic susceptibilities as a function of the Ni-O-Ni angle: $\chi_{1,1}$ is the $d_{x^{2}-y^{2}}$ component and $\chi_{2,2}$ the $d_{z^{2}}$ component. Bottom: spectral weight at the Fermi level as a function of $Q$.

(spinodals) of the BDI and metallic phases, with a narrow coexistence region in-between. Solid lines show stable non-zero solutions, $\bar{Q}[K, W]$. For fixed $K$, the bandwidth $W$ (equivalently, $\chi_{e}$ ) determines whether the PM groundstate is a BDI phase. Variation of $K$ controls smoothly the position of the phase boundaries.

Realistic DFT+DMFT. We now perform ab-initio DFT + DMFT calculations to confirm the physics found in the model calculations and assess materials trends quantitatively. The impurity model is constructed by projecting onto a low-energy $e_{g}$ subspace following the scheme described in Refs. [17, 18] (see [41] for details).

Fig. 3 shows the calculated $\nu(Q)$ for $R=\mathrm{Lu}, \mathrm{Sm}$, and $\operatorname{Pr}$ (top). The overall nonlinear behavior of $\nu(Q)$ is very similar to that in the model calculations (Fig.1), with the non-linearity clearly related to the MIT (Fig. 3 bottom), also indicating a first-order character of the $\mathrm{BD} / \mathrm{MIT}$. This confirms that the model indeed incorporates the essential underlying physics. Furthermore, we obtain a strong decrease of the amplitude of the non-linearity in $\nu(Q)$ from $R=\mathrm{Lu}$ towards $R=\mathrm{Pr}$, consistent with the bandwidth variation in the model. The $d_{x^{2}-y^{2}}$ component of the electronic susceptibility displayed in Fig. 3 (top, inset) increases steadily as the Ni-O-Ni bond angle is reduced (i.e., for for smaller $R$ ions). This shows that the stronger octahedral rotations for $R=\mathrm{Lu}$ compared to 
$R=\operatorname{Pr}$ lead to an increased $\chi_{e}[27,38$, and thus control directly the electronic instability.

Finally, the values obtained for $K$ and $g$ from DFT lead to stable equilibrium BM amplitudes for all investigated compounds. The value obtained for $\bar{Q}$ for $\mathrm{LuNiO}_{3}$ of $0.073 \AA$ is in very good agreement with available experimental data $\left(Q_{\exp }=0.075 \AA[26]\right) . \mathrm{PrNiO}_{3}$ seems to be very close to the transition, as its $\bar{Q}$ value is very close to the MIT, and the stable BM would eventually be lost if a reduced $U$ is used for $\mathrm{PrNiO}_{3}$, as suggested by our cRPA calculations [22. Moreover, previous studies find that the magnetic order appears to be crucial in stabilizing the $\mathrm{BD}$ phase in $\mathrm{PrNiO}_{3}$ and $\mathrm{NdNiO}_{3}$ [7, 22 24. The stability and influence of the magnetic order goes beyond the scope of our work and requires further investigation. However, the overall trend of an increase in $Q$ and in the stability of the BM through the series for smaller $R$-cations is consistent with experiments and in line with earlier studies [19, 22, 32.

Landau theory. We finally show that the main qualitative features of the MIT found above can be rationalized in terms of a Landau theory, which involves two coupled scalar order parameters (OP): the ED, $\nu$, and an additional OP, $\phi$, which distinguishes between metallic (conventionally associated with $\phi>0$ ) and insulating behavior $(\phi<0)$. The reason why this second OP is required is clear from the results above: a non-zero value of $\nu$ can correspond either to a metallic phase (at small values of the on-site modulation, $\Delta_{s}$, or, equivalently, of $Q$ ), or to an insulating one. In other words, a metallic monoclinic phase with a non zero-value of the BM amplitude $Q$ is in principle possible, in agreement with recent experimental findings [43]. Such an OP has been introduced to describe the Mott transition in the DMFT framework, in analogy to the liquid-gas transition [44, 45]. Note that the present Landau theory aims at describing the MIT between the two PM phases, while the earlier Landau descriptions [23, 38] aimed at the magnetic transition (see also Ref. [5] in relation to ruthenates.)

Assuming the simplest coupling allowed by symmetry, $\phi \nu^{2}$, the PM transitions can be described by the following energy functional: $F[\nu, \phi]=F_{\nu}+F_{\phi}+\lambda \phi \nu^{2}$, with $F_{\nu}=$ $\frac{1}{2} b \nu^{2}+\frac{1}{4} c \nu^{4}-\Delta_{s} \nu+\frac{1}{2} \kappa \Delta_{s}^{2}, F_{\phi}=\frac{1}{2} a \phi^{2}+\frac{1}{4} u \phi^{4}-h \phi$, where $u, c>0$ and the coupling parameter $\lambda>0$ is of order one. The coupling to the lattice is represented by the linear term $\Delta_{s} \nu$, with $\Delta_{s}$ serving as a symmetry-breaking field (alternatively, the BM can be introduced with $Q=$ $\left.g \Delta_{s}\right)$. In the absence of ED, the system is a metal, so that we must assume $a$ and $h$ to be positive. $b$ is a key control parameter related to $\chi_{e}$, and it depends critically on external parameters such as, e.g., the bandwidth, $b=$ $b_{0}\left(W-W_{c}\right)$.

Without loss of generality we can set $u=c=a=1$. Minimizing $F[\nu, \phi]$ yields the following coupled equations

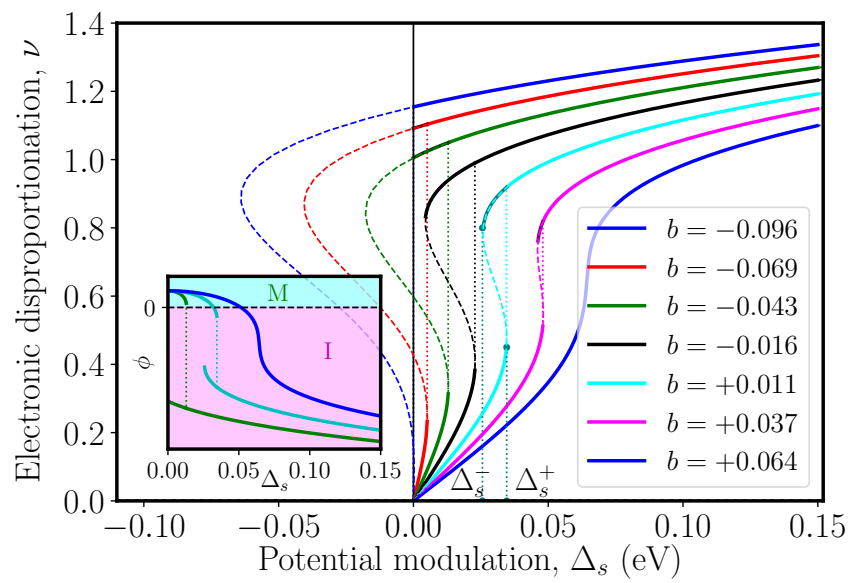

FIG. 4. ED, $\nu$, as a function of $\Delta_{s}$ for various values of parameter $b$, as obtained within the Landau theory described in the text. Dashed lines display unstable solutions. Critical points $\Delta_{s}^{-}, \Delta_{s}^{+}$are indicated for one of the cases. Inset: MI OP $\phi$ as a function of $\Delta_{s}$ for three selected cases $(b=-0.043$, $0.011,0.064)$. Region marked with "M": metallic phase $(\phi>$ $0)$; region marked with "I": insulating phase $(\phi<0)$.

of state:

$$
\begin{aligned}
& b \nu+\nu^{3}+2 \lambda \phi \nu=\Delta_{s} \\
& \phi+\phi^{3}=h-\lambda \nu^{2}
\end{aligned}
$$

The numerical solution of these equations is displayed in Fig. 4 for varying $b$. For $b>0$, starting from a value $\phi=\phi_{0}>0$, typical for the metallic phase at $\nu=0$, and increasing $\Delta_{s}$ leads to a strongly non-linear dependence of $\nu$ on $\Delta_{s}$, with $\phi$ continuously decreasing (because of the $-\lambda \nu^{2}$ term) and gradually reaching negative values (inset of Fig. 4). At a critical value of $b$, the $\nu\left[\Delta_{s}\right]$ curves acquire a vertical tangent and beyond this value, an S-shape with an unstable branch is found, typical of a first-order transition, with two vertical tangents delimiting the two spinodal values of $\Delta_{s}, \Delta_{s}^{-}$and $\Delta_{s}^{+}$. When $b$ is further decreased, a spontaneous instability is found, with a jump of $\nu$ to a finite value for an infinitesimal $\Delta_{s}$. This general behavior is in excellent qualitative agreement with Fig. 1. A more detailed analysis (see [41) reveals that both spinodal values scale as $\Delta_{s}^{*} \sim\left(b-b^{*}\right)^{\frac{3}{2}} \sim\left(W-W^{*}\right)^{\frac{3}{2}}$, which is apparent in Fig. 2 .

Conclusions. We have presented a theory of the combined structural and electronic MIT in bulk $R \mathrm{NiO}_{3}$. The driving force is the proximity to the electronic disproportionation instability, which is cooperatively reinforced by the coupling to the lattice breathing mode. The transition is thus controlled both by the electronic charge susceptibility and by the stiffness of this mode. The key non-linearities associated with this cooperative effect can be rationalized in terms of a Landau theory. Our work provides a pathway to understanding the MIT in other geometries, such as ultrathin films and heterostructures, 
and is likely to have general applicability to other materials with a strong interplay between electronic correlations and lattice degrees of freedom.

We are grateful to C. Ahn, D. Basov, J. Chakhalian, A. Georgescu, A. J. Millis, J. M. Triscone, D. van der Marel for discussions. This work was supported by the European Research Council (ERC319286-'QMAC'), the Swiss National Science Foundation (NCCR MARVEL), the Swiss National Supercomputing Centre (projects s575, s624, mr17, s820), and the Forschungsförderungsgesellschaft (FFG) COMET program IC-MPPE (project No 859480). The Flatiron Institute is a division of the Simons Foundation.

[1] P. Zubko, S. Gariglio, M. Gabay, P. Ghosez, and J.M. Triscone, Annu. Rev. Condens. Matter Phys. 2, 141 (2011).

[2] D. B. McWhan, T. M. Rice, and J. P. Remeika, Phys. Rev. Lett. 23, 1384 (1969)

[3] Y. Tokura, Rep. Prog. Phys. 69, 797 (2006)

[4] S. Nakatsuji, S. ichi Ikeda, and Y. Maeno, J. Phys. Soc. Jpn. 66, 1868 (1997)

[5] Q. Han and A. Millis, Phys. Rev. Lett. 121, 067601 (2018)

[6] J. B. Torrance, P. Lacorre, A. I. Nazzal, E. J. Ansaldo, and C. Niedermayer, Phys. Rev. B 45, 8209 (1992).

[7] M. L. Medarde, J. Phys.: Condens. Matter 9, 1679 (1997).

[8] G. Catalan, Phase Transit. 81, 729 (2008)

[9] S. Catalano, M. Gibert, J. Fowlie, J. Iñiguez, J.-M. Triscone, and J. Kreisel, Rep. Prog. Phys. 81, 046501 (2018)

[10] S. Middey, J. Chakhalian, P. Mahadevan, J. W. Freeland, A. J. Millis, and D. D. Sarma, Annu. Rev. Mater. Res. 46, 305 (2016).

[11] J. M. Rondinelli and N. A. Spaldin, Adv. Mater. 23, 3363 (2011).

[12] A. Demourgues, F. Weill, B. Darriet, A. Wattiaux, J. Grenier, P. Gravereau, and M. Pouchard, Journal of Solid State Chemistry 106, 330 (1993).

[13] T. Mizokawa, D. I. Khomskii, and G. A. Sawatzky, Phys. Rev. B 61, 11263 (2000).

[14] S. Johnston, A. Mukherjee, I. Elfimov, M. Berciu, and G. A. Sawatzky, Phys. Rev. Lett. 112, 106404 (2014).

[15] B. Mandal, S. Sarkar, S. K. Pandey, P. Mahadevan, C. Franchini, A. J. Millis, and D. D. Sarma, ArXiv eprints (2017), arXiv:1701.06819 [cond-mat.str-el]

[16] I. I. Mazin, D. I. Khomskii, R. Lengsdorf, J. A. Alonso, W. G. Marshall, R. M. Ibberson, A. Podlesnyak, M. J. Martínez-Lope, and M. M. Abd-Elmeguid, Phys. Rev. Lett. 98, 176406 (2007).

[17] A. Subedi, O. E. Peil, and A. Georges, Phys. Rev. B 91, 075128 (2015).

[18] P. Seth, O. E. Peil, L. Pourovskii, M. Betzinger, C. Friedrich, O. Parcollet, S. Biermann, F. Aryasetiawan, and A. Georges, Phys. Rev. B 96, 205139 (2017).

[19] J. Varignon, M. N. Grisolia, J. Íñiguez, A. Barthélémy, and M. Bibes, npj Quantum Materials 2 (2017), 10.1038/s41535-017-0024-9

[20] H. Park, A. J. Millis, and C. A. Marianetti, Phys. Rev. Lett. 109, 156402 (2012).

[21] H. Park, A. J. Millis, and C. A. Marianetti, Phys. Rev. B 89, 245133 (2014)

[22] A. Hampel, P. Liu, C. Franchini, and C. Ederer, npj Quantum Materials 4, 5 (2019)

[23] J. Ruppen, J. Teyssier, I. Ardizzone, O. E. Peil, S. Catalano, M. Gibert, J.-M. Triscone, A. Georges, and D. van der Marel, Phys. Rev. B 96, 045120 (2017).

[24] K. Haule and G. L. Pascut, Sci. Rep. 7, 10375 (2017).

[25] K. W. Post, A. S. McLeod, M. Hepting, M. Bluschke, Y. Wang, G. Cristiani, G. Logvenov, A. Charnukha, G. X. Ni, P. Radhakrishnan, M. Minola, A. Pasupathy, A. V. Boris, E. Benckiser, K. A. Dahmen, E. W. Carlson, B. Keimer, and D. N. Basov, Nat. Phys. 14, 1056 (2018)

[26] J. A. Alonso, M. J. Martínez-Lope, M. T. Casais, J. L. García-Muñoz, M. T. Fernández-Díaz, and M. A. G. Aranda, Physical Review B 64, 094102 (2001)

[27] Y. Lu, Z. Zhong, M. W. Haverkort, and P. Hansmann, Phys. Rev. B 95, 195117 (2017)

[28] G. G. Guzmán-Verri, R. T. Brierley, and P. B. Littlewood, ArXiv e-prints (2017), arXiv:1701.02318 [condmat.str-el]

[29] A. Mercy, J. Bieder, J. Íñiguez, and P. Ghosez, Nat. Comm. 8, 1677 (2017).

[30] C. Girardot, J. Kreisel, S. Pignard, N. Caillault, and F. Weiss, Phys. Rev. B 78, 104101 (2008)

[31] S. Prosandeev, L. Bellaiche, and J. Íniguez, Phys. Rev. B 85, 214431 (2012).

[32] A. Hampel and C. Ederer, Phys. Rev. B 96, 165130 (2017)

[33] J. Ruppen, J. Teyssier, O. E. Peil, S. Catalano, M. Gibert, J. Mravlje, J.-M. Triscone, A. Georges, and D. van der Marel, Phys. Rev. B 92, 155145 (2015).

[34] Z. Liao, N. Gauquelin, R. J. Green, K. Müller-Caspary, I. Lobato, L. Li, S. V. Aert, J. Verbeeck, M. Huijben, M. N. Grisolia, V. Rouco, R. E. Hage, J. E. Villegas, A. Mercy, M. Bibes, P. Ghosez, G. A. Sawatzky, G. Rijnders, and G. Koster, Proc. Natl. Acad. Sci. U.S.A 115, 9515 (2018).

[35] A. B. Georgescu, O. E. Peil, A. Disa, A. Georges, and A. J. Millis, ArXiv e-prints (2018), 1810.00480

[36] A. Georges, G. Kotliar, W. Krauth, and M. J. Rozenberg, Rev. Mod. Phys. 68, 13 (1996).

[37] G. Kotliar, S. Y. Savrasov, K. Haule, V. S. Oudovenko, O. Parcollet, and C. A. Marianetti, Rev. Mod. Phys. 78, 865 (2006)

[38] S. B. Lee, R. Chen, and L. Balents, Phys. Rev. B 84, 165119 (2011); idem., Phys. Rev. Lett. 106, 016405 (2011)

[39] A. Georges, L. D. Medici, and J. Mravlje, Annu. Rev. Condens. Matter Phys. 4, 137 (2013).

[40] We checked that in all our DMFT calculations the orbital occupation numbers always ended up being nearly equal.

[41] Supplemental Materials.

[42] S. Catalano, M. Gibert, V. Bisogni, F. He, R. Sutarto, M. Viret, P. Zubko, R. Scherwitzl, G. A. Sawatzky, T. Schmitt, and J.-M. Triscone, APL Mater. 3, 062506 (2015)

[43] S. Middey, D. Meyers, M. Kareev, Y. Cao, X. Liu, P. Shafer, J. W. Freeland, J.-W. Kim, P. J. Ryan, and 
J. Chakhalian, Phys. Rev. Lett. 120, 156801 (2018)

[44] G. Kotliar, E. Lange, and M. J. Rozenberg, Phys. Rev. Lett. 84, 5180 (2000)
[45] P. Limelette, A. Georges, D. Jérome, P. Wzietek, P. Metcalf, and J. M. Honig, Science 302, 89 (2003). 